\title{
Bcl-2 inhibe-t-il l'apoptose en s'opposant à l'action des radicaux oxygènes?
}

La protéine $\mathrm{Bcl}-2$, équivalent chez les mammifères du produit du gène anti-apoptotique ced 9 de Caenorhabditis elegans, joue un rôle essentiel dans le contrôle négatif de l'apoptose [1]. A la fin de l'année 1993, deux équipes sont parvenues à créer des lignées de souris [2] ou des populations de cellules [3] totalement déficientes en $\mathrm{Bcl}-2$ à la suite d'une interruption par recombinaison homologue de la continuité des deux allèles de ce gène. Veis $e t$ al. de Saint-Louis (MO, USA) ont montré que la mutation homozygote du gène $b c l$-2 n'empêchait pas un développement embryonnaire normal et complet, mais aboutissait à un retard de croissance et à une mortalité postnatale précoce. Le système hématopoïétique, notamment les lymphocytes, se développe normalement. Cependant, la rate et le thymus subissent une involution rapide secondaire à une apoptose massive. En culture, les thymocytes ont une susceptibilité très augmentée aux glucocorticoïdes et aux radiations ionisantes. Les reins sont massivement polykystiques, ce qui entraîne une insuffisance rénale sévère. De manière singulière, la pigmentation du pelage change rapidement, virant d'une couleur noire ou agouti initiale, selon les cas, au gris [2]. Nakayama et al. de SaintLouis (MO, USA) et Boston (MA, USA) étudièrent, quant à eux, les conséquences de l'invalidation des deux allèles $b c l-2$ par la méthode du double knock-out suivi de la création de chimères. Dans cette approche, des cellules ES sont modifiées au niveau des deux allèles du gène $b c l-2$ et sont réintroduites dans des blastocystes, engendrant des animaux chimères dont certaines cellules sont

tes $\mathrm{T}$ et $\mathrm{B} b c \mathrm{l}-2 \%$ disparaissent de la moelle osseuse, du thymus et du sang périphérique à la quatrième semaine de vie. Ex vivo, la sensibilité des lymphocytes $\mathrm{T}$ déficients en Bcl-2 à l'induction de l'apoptose par les radiations ionisantes et les glucocorticoïdes est notée par ces auteurs, comme par les précédents [3].

L'apparition de touffes de poils gris chez les souris $b c l-2^{-} \%$ évoque une sensibilité particulière des précurseurs de la mélanine à l'oxydation au niveau des follicules pileux. En effet, l'un des précurseurs de la mélanine, la DOPA-quinone, est extrêmement sensible à l'oxydation. Hockenbery, de Seattle (WA, USA) associé à l'équipe ayant créé les souris $b c-2 \digamma$, démontre que la protéine Bcl-2 inhibe la peroxydation des lipides engendrée par le peroxyde d'hydrogène $\mathrm{H}_{2} \mathrm{O}_{2}$ et par d'autres peroxydes [4]. Ces auteurs ont étudié une lignée cellulaire dont l'apoptose est prévenue par l'interleukine-3. Dans ces cellules, la ménadione, entraînant la production d'anion superoxyde, et l' $\mathrm{H}_{2} \mathrm{O}_{2}$ sont de puissants inducteurs de l'apoptose qui peut être prévenue par l'hyper-expression de $b c l-2$. L'introduction dans ces cellules d'un vecteur d'expression pour la superoxyde dismutase ne les protège pas contre l'apoptose, alors qu'un vecteur commandant la synthèse de glutathion peroxydase a un net effet protecteur. La glutathion peroxydase détruit $\mathrm{l}^{\prime} \mathrm{H}_{2} \mathrm{O}_{2}$ aux dépens de l'oxydation du glutathion réduit. Ces résultats suggèrent que l'apoptose est relayée par les peroxydes, probablement par l'intermédiaire du radical hydroxyle $\mathrm{OH}^{\circ}$. Bcl-2 ne diminue pas la production d' $\mathrm{H}_{2} \mathrm{O}_{2}$ mais protège les lipides de la peroxydation. La localisation majoritaire de
Bcl-2 à la membrane des mitochondries, site majeur de production d'anion superoxyde qui sera transformé en peroxyde d'hydrogène par la superoxyde dismutase (SOD), est en accord avec un rôle protecteur assuré par Bcl-2 contre les effets nocifs des radicaux actifs d'oxygène engendrés à partir de l'anion $\mathrm{O}_{2}{ }^{-}$. Un semblable effet de protection par Bcl-2 contre les agressions oxydatives a été observé par Kane et al. de Los Angeles (CA, USA) dans la lignée cellulaire neuronale d'origine hypothalamique GT1-7 [5]. Au niveau de ces cellules, l'inhibition de la synthèse du glutathion réduit GSH ou sa destruction entraine une mort cellulaire rapide qui est évitée lorsque les cellules synthétisent une grande quantité de $\mathrm{Bcl}-2$ grâce à l'introduction d'un vecteur d'expression. Les expériences suggèrent que Bcl-2 pourrait s'opposer à l'effet toxique des radicaux hydroxyles $\mathrm{OH}^{\circ}$ engendrés à partir de $\mathrm{l}^{\prime} \mathrm{H}_{2} \mathrm{O}_{2}$ en présence de fer ferreux $\left(\mathrm{Fe}^{2+}\right)$. L'intérêt tout particulier de cette découverte que $\mathrm{Bcl}-2$ pourrait s'opposer aux effets toxiques de radicaux oxygènes vient du fait que l'on soupçonnait depuis longtemps l'intervention des dommages oxydatifs dans de nombreux phénomènes de sénescence et de neurodégénérescence [6]. Notamment, de nombreuses données suggèrent que la cytotoxicité du glutamate, ou d'autres substances, pourrait passer par l'activation de protéases susceptibles de convertir la xanthine déshydrogénase en xanthine oxydase, enzyme produisant de l'anion superoxyde qui sera transformé en $\mathrm{H}_{2} \mathrm{O}_{2}$ par les superoxydes dismutases [6]. D'ailleurs, des mutations du gène de la superoxyde dismutase cytoplasmique à $\mathrm{Cu}^{2+}$ et à $\mathrm{Zn}^{2+}$ ont été décou- 
vertes dans des formes familiales et quelques formes sporadiques de sclérose latérale amyotrophique $(\mathrm{m} / \mathrm{s}$ $n^{\circ}$ 4, vol. 9, p. 469; $n^{\circ}$ 5, vol. 9, p. 643). De nombreux exemples existent où l'apoptose de cellules en l'absence de facteurs de croissance, de facteurs neurotrophiques ou de cytokines peut être évitée par une synthèse accrue de Bcl-2, suggérant que cette protéine anti-apoptotique pourrait relayer au moins certains des effets de ces facteurs [1, 7]. Selon cette vision, plusieurs mécanismes intrinsèques ou plusieurs types de réponse à des stimulations extrinsèques pourraient aboutir à une voie commune d'apoptose mettant en jeu l'altération de constituants cellulaires par des radicaux oxygènes actifs, notamment le radical hydroxyle $\mathrm{OH}^{\circ}$. Cette production de dérivés toxiques de l'oxygène pourrait être accrue dans certaines maladies génétiques ou dégénératives. Symétriquement, ces maladies pourraient être liées à l'inefficacité des systèmes biologiques de protection contre ces radicaux oxygènes, systèmes où $\mathrm{Bcl}-2$ serait un acteur essentiel. De telles anomalies pourraient intervenir à une étape quelconque de la voie de transmission du signal entre des récepteurs membranaires de facteurs de survie et le gène $b c l$ - 2 .

Peu de choses sont aujourd'hui connues des mécanismes de la régulation de l'expression du gène $b c l-2$ et de son couplage avec des récepteurs membranaires. Cependant, Fernandez-Tarabia et Bischoff ont récemment cloné l'ADNc codant pour un partenaire de $\mathrm{Bcl}-2$ qui est une protéine de la famille Ras liant le GTP [8]. Pour ce faire, ces auteurs de Richmond, CA, USA, ont utilisé la technique des doubles hybrides chez la levure $\left(m / s n^{\circ} 2\right.$, vol. 10, p. 206). La molécule capable de former un complexe avec Bcl-2 est R-ras p23, une protéine de 23 $\mathrm{kDa}$ à $55 \%$ similaire à H-ras p21. Contrairement aux protéines p2l ras, p23r-ras n'a pas de pouvoir transformant. On peut faire l'hypothèse que cette molécule est un transducteur d'un signal contrôlant l'activité ou les effets de Bcl-2.

Les mécanismes de protection contre les dérivés actifs de l'oxygène sont très probablement ancestraux puisqu’ils ont dû apparaître dès l'accumulation de l'oxygène dans l'atmosphère terrestre. Par conséquent, la régulation par $\mathrm{Bcl}-2$ ou par des molécules homologues de l'apoptose que peuvent induire les dommages oxydatifs pourrait être également un processus extrêmement ancien, continuant de jouer un rôle essentiel dans la survie des cellules des organismes pluricellulaires développés

\section{RÉFÉRENCES}

1. Kahn A, Briand P. L'apoptose, une mort programmée ou une prolifération avortée? médecine/sciences $1993 ; 9: 663-5$.

2. Veis D, Sorenson CM, Shutter JR, Korsmeyer SJ. Bcl-2 deficient mice demonstrate fulminant lymphoid apoptosis, polycystic kidneys and hypopigmented hair. Cell $1993 ; 75: 229-240$.

3. Nakayama K-1, Nakayama K, Negishi I, Kuida K, Shinkai Y', Louie MC, Fields IE, Lucas PJ, Stewart V, Alt FW, Loh DY. Disappearance of the lymphoid system in Bcl-2 homozygous mutant chimeric mice. Sicience $1993 ; 261: 1584-8$.

4. Hockenbery DM, Oltvai ZN, Yin X-M, Milliman CL, Korsmeyer SJ. Bcl-2 functions in an antioxydant pathway to prevent apoptosis. Cell 1993; 75 : 241-51

5. Kane DJ, Sarafian TA, Anton R, Hahn H, Gralla EB, Valentine JS, Ord T, Bredesen DE. Bcl-2 inhibition of neural death decreased generation of reactive oxygen species. Science 1993; 262: 1274-7.

6. Coyle JT, Puttfarcken P. Oxidative stress, glutamate and neurodegenerative disorders. Science 1993; 262: 689-95.

7. Raff MC, Barres BA, Burne JF, Coles HS, Ishizaki Y, Jacobson MD. Programmed cell death and the control of cell survival : lessons from the nervous system. Science 1993 262: $695-700$

8. Fernandez-Sarabia MJ, Bischoff JR. Bcl-2 associates with the ras-related protein R-ras p23. Nature $1993 ; 366$ : 274-5

\section{Axel Kahn}

Directeur de l'unité de recherches en génétique et pathologie moléculaires, Inserm U 129. ICGM, 24, rue du Faubourg Saint-Jacques, 75014 Paris, France.

TIRÉS A PART

A. Kahn. 